\title{
О ДВУХ ЗАДАЧАХ ТЕОРИИ ЧАСТИЧНО ТОТАЛЬНО КОМПОЗИЦИОННЫХ ФОРМАЦИЙ КОНЕЧНЫХ ГРУПП
}

\author{
В. В. Щербина
}

Статъя представлена членом редакционной коллегии В. Б. Василъевым

\author{
Государственное учреждение образования «Институт подготовки научных кадров \\ Национальной академии наук Беларуси», \\ г. Минск, 220049, Республика Беларусь \\ E-mail: shcherbinavv@tut.by
}

\begin{abstract}
Аннотация. В работе рассматриваются только конечные группы. Изучаются свойства решетки всех функторно замкнутых частично тотально композиционных формаций. Доказано, что для любого подгруппового функтора $\tau$ решетка $c_{\omega_{\infty}}^{\tau}$ всех $\tau$-замкнутых тотально $\omega$-композиционных формаций является алгебраической. Кроме того, установлена индуктивность данной решетки. В качестве следствия основного результата установлены алгебраичность и индуктивность решетки $c_{p_{\infty}}^{\tau}$ всех $\tau$-замкнутых тотально $p-$ композиционных формаций, а также решетки $c_{\infty}^{\tau}$ всех $\tau$-замкнутых тотально композиционных формаций.

Ключевые слова: конечная группа, формация групп, тотально $\omega$-композиционная формация, решетка формаций, $\tau$-замкнутая формация, алгебраическая решетка, индуктивная решетка.
\end{abstract}

Для цитирования: Щербина В. В. 2020. О двух задачах теории частично тотально композиционных формаций конечных групп. Прикладная математика \& Физика. 52(1): 18-32.

DOI 10.18413/2687-0959-2020-52-1-18-32.

\section{ON TWO PROBLEMS OF THE THEORY OF PARTIALLY TOTALLY COMPOSITION FORMATIONS OF FINITE GROUPS}

\author{
V. V. Shcherbina \\ Article submitted by a member of the editorial board V. B. Vasilyev \\ State Institution of Education «Graduate School of the National Academy of Sciences of Belarus», \\ Minsk, 220049, Republic of Belarus \\ E-mail: shcherbinavv@tut.by
}

Received December 25, 2019

\begin{abstract}
All groups considered in the paper are assumed to be finite. Further, $\omega$ denotes some nonempty set of primes, and $\tau$ is a subgroup functor in the sense of A.N. Skiba. Recall that a formation is a class of groups that is closed under taking homomorphic images and finite subdirect products. The paper studies properties of the lattice of all closed functorially totally partially saturated formations. We prove that for any subgroup functor $\tau$, the lattice $c_{\omega_{\infty}}^{\tau}$ of all $\tau$-closed totally $\omega$-composition formations is algebraic. Furthermore, we prove that the lattice mentioned above is inductive. In particular, we show that the lattice $c_{p_{\infty}}^{\tau}$ of all $\tau$-closed totally $p$-composition formations and the lattice $c_{\infty}^{\tau}$ of all $\tau$-closed totally composition formations are both algebraic and inductive. Thus, new classes of algebraic and inductive lattices of formations are found.

Key words: finite group, formation of groups, totally $\omega$-composition formation, lattice of formations, $\tau$-closed formation, algebraic lattice, inductive lattice.

For citation: Shcherbina V. V. 2020. On two problems of the theory of partially totally composition formations of finite groups. Applied Mathematics \& Physics. 52(1): 18-32 (in Russian).
\end{abstract}

DOI 10.18413/2687-0959-2020-52-1-18-32.

Введение. В работе рассматриваются только конечные группы. Мы будем использовать терминологию, принятую в работах Л. А. Шеметкова, А. Н. Скибы и других авторов [Шеметков, 1978; Шеметков, Скиба, 1989; Doerk, Hawkes, 1992; Скиба, 1997; Скиба, Шеметков, 2000; Воробьев, 2012].

Понятие формации было введено В. Гашюцом [Gaschuts, 1963] в связи с разработкой общих методов отыскания подгрупп в конечных разрешимых группах. Класс конечных групп называют формацией, если он замкнут относительно взятия гомоморфных образов и конечных подпрямых произведений. В той же работе В. Гашюца был впервые выделен важный для приложений класс насыщенных формаций и предложен способ конструирования такого рода формаций при помощи 
специальных функций. Установленная А. Н. Скибой [1986] модулярность решетки всех формаций, а также решетки всех насыщенных формаций, стала основой в использовании решеточных методов для решения многих открытых вопросов теории формаций конечных групп. Основные результаты структурной теории формаций изложены в книгах Л. А. Шеметкова, А. Н. Скибы и других авторов [Шеметков, Скиба, 1989; Doerk, Hawkes, 1992; Скиба, 1997; Guo, 2000; Ballester-Bolinches, Ezquerro, 2006; Воробьев, 2012]. Ряд свойств решетки всех тотально насыщенных формаций установлен в работах А. Н. Скибы, Н. Н. Воробъева и других авторов [Скиба, 1997; Воробьев, 2000; Сафонов, 2007; Сафонов, 2008; Сафонов, 2010]. Изучению свойств решетки всех тотально композиционных формаций посвящены работы А. А. Царева [Tsarev, 2018a; Tsarev, 2019].

В 1999 году в теории формаций А. Н. Скибой и Л. А. Шеметковым был предложен подход, использующий идеи частичной [Шеметков, 1984] и кратной (тотальной) [Скиба, 1987] насыщенности формации, объединенные в понятии $n$-кратно (тотально) $\omega$-насыщенной формации [Скиба, Шеметков, 1999]. Дальнейшим развитием идей и результатов теории формаций явилось перенесение данного подхода на более широкий класс частично композиционньх формаций [Скиба, Шеметков, 2000]. Особая роль частично тотально насыщенных (композиционных) формаций обусловлена прежде всего тем, что большинство наиболее известных конкретных классов конечных групп являются частично тотально насыщенными (композиционными) формациями, и поэтому они наиболее часто применяются в различных приложениях теории классов конечных групп. Изучение ряда свойств решетки частично тотально насыщенных формаций проведено в работах В. Г. Сафонова и других авторов [Сафонов, 2004; Сафонов, Сафонова, 2014; Сафонов, Сафонова, 2017; Щербина, Сафонов, 2019а, б]. Тем не менее в настоящее время решетка (частично) тотально композиционных формаций является одной из менее изученных решеток формаций конечных групп. Об этом свидетельствует ряд открытых вопросов, поставленных в работах А. Н. Скибы, Л. А. Шеметкова и других авторов [Скиба, Шеметков, 2000; Skiba, Vorob'ev, 2013; Tsarev, Vorob'ev, 2018]. В теории частично композиционных формаций А. Н. Скибой и Л. А. Шеметковым [2000] была установлена модулярность и алгебраичность решетки всех $n$-кратно $\mathfrak{L}$-композиционных формаций при любом натуральном $n$, а также поставлена следующая проблема: алгебраична ли решетка $c_{\infty}^{\mathfrak{L}}$ всех тотально L-композиционных формаций (см. проблема 1 [Скиба, Шеметков, 2000]).

В совместной работе А. А. Царева и Н. Н. Воробьева [Tsarev, Vorob'ev, 2018] поставлены вопросы об алгебраичности и индуктивности решетки $c_{\omega_{\infty}}^{\tau}$ всех $\tau$-замкнутых тотально $\omega$-композиционных формаций (см. вопрос 5.5(1) и (2) [Tsarev, Vorob'ev, 2018]).

В данной работе мы даем положительные ответы на эти вопросы: доказано, что решетка $c_{\omega_{\infty}}^{\tau}$ всех $\tau$-замкнутых тотально $\omega$-композиционных формаций алгебраична (теорема 3.1 ), а также установлена индуктивность указанной решетки (теорема 2.2).

1. Предварительные сведения. В дальнейшем $\omega, \pi$ обозначают некоторые непустые множества простых чисел, $p$ и $q$-некоторые простые числа, $K \lambda A$-полупрямое произведение группы $K$ с некоторой группой операторов $A$ этой группы. Для каждого множества простых чисел $\pi$ через $\pi^{\prime}$ обозначается множество $\mathbb{P} \backslash \pi$, где $\mathbb{P}$-множество всех простых чисел. Символ $\pi(G)$ обозначает множество всех различных простых делителей порядка группы $G, \pi(\mathfrak{X})$-объединение множеств $\pi(G)$ для всех групп $G$ из $\mathfrak{X}$. Для произвольного класса групп $\mathfrak{F} \supseteq(1)$ символ $G^{\mathfrak{F}}$ обозначает пересечение всех таких нормальных подгрупп $N$, что $G / N \in \mathfrak{F}$, символ $G_{\mathfrak{F}}$-произведение всех нормальных $\mathfrak{F}$ подгрупп группы $G$. Символом (1) обозначается класс всех единичных групп. Символы $\mathfrak{G}, \mathfrak{G}_{\pi}, \mathfrak{N}_{p}$, $\mathfrak{S}_{\pi}$ и $\mathfrak{N}_{\pi}$ обозначают класс всех групп, $\pi$-групп, $p$-групп, разрешимых $\pi$-групп и нильпотентных $\pi$-групп соответственно. Если $\pi=\varnothing$, то, по определению, $\mathfrak{G}_{\varnothing}=\mathfrak{N}_{\varnothing}=\mathfrak{S}_{\varnothing}=(1)$.

Полагают

$$
O_{p}(G)=G_{\mathfrak{N}_{p}}, \quad O_{\pi}(G)=G_{\mathfrak{G}_{\pi}} \quad R_{\omega}(G)=G_{\mathfrak{S}_{\omega}}, \quad F_{p}(G)=G_{\mathfrak{G}_{p^{\prime}} \mathfrak{N}_{p}} .
$$

Символ $C^{p}(G)$ обозначает пересечение централизаторов всех тех главных факторов группы $G$, чьи композиционные факторы имеют простой порядок $p$ (если в группе $G$ нет таких факторов, то полагают $\left.C^{p}(G)=G\right)$.

Неединичная группа $G$ называется монолитической, если в ней имеется лишь одна минимальная нормальная подгруппа (монолит группы $G$ ). Для произвольной совокупности групп символ $(\mathfrak{X})$ обозначает абстрактное замыкание $\mathfrak{X}$, т. е. класс всех групп, изоморфных группам из $\mathfrak{X}$. Символы $\mathcal{K}(\mathfrak{X})$ и $\operatorname{Com}(\mathfrak{X})$ обозначают класс всех тех простых групп и соответственно класс всех тех простых абелевых групп, которые встречаются в качестве композиционных факторов некоторых групп из $\mathfrak{X}$.

Класс всех простых групп мы обозначаем символом э. Для произвольного класса простых групп $\mathfrak{T}$ через $\mathfrak{T}^{\prime}$ обозначено множество $\mathfrak{I} \backslash \mathfrak{T}$. Для произвольного непустого класса простых групп $\mathfrak{L}$ через $\mathfrak{L}^{+}$обозначена совокупность всех абелевых групп из $\mathfrak{L}$, через $\mathfrak{L}^{-}-$совокупность всех простых неабелевых групп из $\mathfrak{L}$ [Скиба, Шеметков, 2000]. 
Для произвольного класса простых групп $\mathfrak{T}$ символ $E(\mathfrak{T})$ обозначает класс всех таких групп, у которых все композиционные факторы принадлежат т. По определению, единичные группы принадлежат $E(\mathfrak{T})$.

Пусть $f-$ произвольная функция вида

$$
f: \omega \cup\left\{\omega^{\prime}\right\} \rightarrow\{\text { формации групп }\},
$$

где $f\left(\omega^{\prime}\right) \neq \varnothing($ формационная $\omega$-функция).

Следуя [Скиба, Шеметков, 2000], сопоставим функции $f$ класс групп

$$
C F_{\omega}(f)=\left(G \mid G / R_{\omega}(G) \in f\left(\omega^{\prime}\right) \text { и } G / C^{p}(G) \in f(p) \text { для всех простых } \pi(\operatorname{Com}(G)) \cap \omega\right) .
$$

Для любой функции $f$ вида $(1)$ класс $C F_{\omega}(f)$ является формацией. Если формация $\mathfrak{F}$ такова, что $\mathfrak{F}=C F_{\omega}(f)$ для некоторой функции вида $(1)$, то $\mathfrak{F}$ называется $\omega$-композиционной или разрешимо $\omega$-насыщенной формацией с $\omega$-композиционным спутником $f$ [Скиба, Шеметков, 2000]. Если при этом все значения $f$ лежат в $\mathfrak{F}$, то $f$ называется внутренним (или приведенным) спутником.

Пусть $\left\{f_{i} \mid i \in I\right\}$-произвольный набор $\omega$-композиционных спутников. Через $\bigcap_{i \in I} f_{i}$ обозначают такой $\omega$-композиционный спутник $f$, что $f(a)=\bigcap_{i \in I} f_{i}(a)$ для всех $a \in \omega \cup\left\{\omega^{\prime}\right\}$. Пусть $f$ и $h-$ $\omega$-композиционные спутники. Тогда полагают $f \leqslant h$, если $f(a) \subseteq h(a)$ для всех $a \in \omega \cup\left\{\omega^{\prime}\right\}$.

Согласно замечанию 1 [Скиба, Шеметков, 2000] любая $\omega$-композиционная формация $\mathfrak{F}$ имеет такой $\omega$-композиционный спутник $F$, что $F\left(\omega^{\prime}\right)=\mathfrak{F}$ и $F(p)=\mathfrak{N}_{p} F(p) \subseteq \mathfrak{F}$ для всех $p \in \omega$, и, кроме того, для произвольного внутреннего $\omega$-композиционного спутника $f$ формации $\mathfrak{F}$ имеет место $f \leqslant F$. Спутник $F$ называется каноническим $\omega$-композиционным спутником формации $\mathfrak{F}$.

Пусть $\mathfrak{X}$ - произвольная совокупность групп, $p$-простое число. Тогда полагают (см.[Скиба, Шеметков, 2000]):

$$
\mathfrak{X}\left(C^{p}\right)= \begin{cases}\operatorname{form}\left(G / C^{p}(G) \mid G \in \mathfrak{X}\right), & \text { если } p \in \pi(\operatorname{Com}(\mathfrak{X})) \cap \omega, \\ \varnothing, & \text { если } p \in \mathbb{P} \backslash(\operatorname{Com}(\mathfrak{X})) \cap \omega .\end{cases}
$$

Пусть $A, B$ - группы, $\varphi: A \rightarrow B$ - эпиморфизм, $\Omega$ и $\Sigma$ - некоторые системы подгрупп в $A$ и $B$ соответственно. Тогда через $\Omega^{\varphi}$ обозначается множество $\left\{H^{\varphi} \mid H \in \Omega\right\}$, а через $\Sigma^{\varphi^{-1}}-$ множество $\left\{H^{\varphi^{-1}} \mid H \in \Sigma\right\}$ всех полных прообразов в $A$ всех групп из $\Sigma$.

Пусть $\mathfrak{X}$ - произвольный непустой класс групп и всякой группе $G \in \mathfrak{X}$ сопоставлена некоторая система ее подгрупп $\tau(G)$. Говорят, что $\tau$ - подгрупповой $\mathfrak{X}$-функтор в смысле А. Н. Скибы [1997] (или, иначе, $\tau$-подгрупповой функтор на $\mathfrak{X}$ ), если для всякого эпиморфизма $\varphi: A \rightarrow B$, где $A, B \in \mathfrak{X}$, выполнены включения $(\tau(A))^{\varphi} \subseteq \tau(B),(\tau(B))^{\varphi^{-1}} \subseteq \tau(A)$ и, кроме того, для любой группы $G \in \mathfrak{X}$ имеет место $G \in \tau(G)$. Если $\mathfrak{X}=\mathfrak{G}$ - класс всех групп, то символ $\mathfrak{X}$ опускают и говорят просто о подгрупповом функторе. Через $\mathrm{S}(G)$ обозначают совокупность всех подгрупп группы $G$, через $\mathrm{S}_{n}(G)$ - совокупность всех нормальных подгрупп группы $G$. Подгрупповой функтор $\tau$ называется тривиальным, если $\tau(G)=\{G\}$, единичным, если $\tau(G)=\mathrm{S}(G)$ для любой группы $G$. Класс групп $\mathfrak{F}$ называется $\tau$-замкнутым, если $\tau(G) \subseteq \mathfrak{F}$ для любой группы $G \in \mathfrak{F}$.

Будем рассматривать только такие подгрупповые функторы $\tau$, что для любой группы $G$ все подгруппы, входящие в $\tau(G)$, субнормальны в $G$. Напомним, что решеткой называется частично упорядоченное множество $L$, в котором любые два элемента имеют точную нижнюю грань, обозначаемую $x \wedge y$, и точную верхнюю грань, обозначаемую $x \vee y$ [Биркгоф, 1984, с. 18]. Решетка $L$ называется полной, если любое ее подмножество $X$ имеет в $L$ точные верхнюю и нижнюю грани. Подрешеткой решетки $L$ называется подмножество $Y \subset L$ такое, что если $a \in Y, b \in Y$, то $a \wedge b \in Y$ и $a \vee b \in Y$. Подрешетка решетки сама является решеткой с теми же операциями объединения и пересечения. Элемент $а$ решетки $L$ называется компактным, если из $a \leqslant \vee\left(x_{j} \mid j \in J\right)$ следует $a \leqslant \vee\left(x_{j} \mid j \in F\right)$ для некоторого конечного подмножества $F \subset J$. Решетка $L$ называется алгебраической, если каждый элемент $a \in L$ является объединением компактньх элементов решетки $L$.

Непустую совокупность формаций $\Theta$ называют полной решеткой формаций [Скиба, 1997; Скиба, Шеметков, 2000], если пересечение любой совокупности формаций из $\Theta$ снова принадлежит $\Theta$ и во множестве $\Theta$ имеется такая формация $\mathfrak{F}$, что $\mathfrak{H} \subseteq \mathfrak{F}$ для любой формации $\mathfrak{H} \in \Theta$. Формации из $\Theta$ называют $\Theta$-формациями. Спутник $f$ называется $\Theta$-значным, если все его значения принадлежат

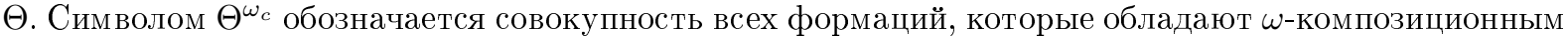
$\Theta$-значным спутником.

Полная решетка формаций $\Theta$ называется частичной алгеброй формаций (см. [Скиба, 1997]), если для любого простого числа $p$ и для любой формации $\mathfrak{F} \in \Theta$ имеет место $\mathfrak{N}_{p} \mathfrak{F} \in \Theta$. Полная решетка формаций $\Theta$ называется индуктивной (см. [Скиба, 1997]), если для любого набора $\left\{\mathfrak{F}_{i} \mid i \in I\right\}$ 
формаций $\mathfrak{F}_{i} \in \Theta^{\omega_{c}}$ и для любого набора $\left\{f_{i} \mid i \in I\right\}$ внутренних $\omega$-композиционных $\Theta$-значных спутников, где $\mathfrak{F}_{i}=C F_{\omega}\left(f_{i}\right)$, имеет место

$$
\vee_{\Theta \omega_{c}}\left(\mathfrak{F}_{i} \mid i \in I\right)=C F_{\omega}\left(\vee_{\Theta}\left(f_{i} \mid i \in I\right)\right) .
$$

А. Н. Скибой введены кратно локальные формации [Скиба, 1987]. Следуя данной концепции, любая формация считается 0-кратно $\omega$-композиционной, а при $n \geqslant 1$ формация $\mathfrak{F}$ называется $n$-кратно $\omega$-композиционной, если $\mathfrak{F}=C F_{\omega}(f)$, где все значения $f$ являются $(n-1)$-кратно $\omega$-композиционным формациями [Скиба, Шеметков, 2000]. Формация $\mathfrak{F}$ называется тотально $\omega$-композиционной, если она $n$-кратно $\omega$-композиционна для всех $n$. Если при этом формация $\mathfrak{F}$ является $\tau$-замкнутой, то $\mathfrak{F}$ называется $\tau$-замкнутой $n$-кратно $\omega$-композиционной и соответственно $\tau$-замкнутой тотально $\omega$-композиционной.

Символом $c_{\omega_{\infty}}^{\tau}$ обозначают совокупность всех $\tau$-замкнутых тотально $\omega$-композиционных формаций. Если $\omega=\mathbb{P}$, то вместо символа $c_{\mathbb{P}_{\infty}}^{\tau}$ используют символ $c_{\infty}^{\tau}$, обозначая совокупность всех $\tau$-замкнутых тотально композиционных формаций.

Если $\tau$-тривиальный подгрупповой функтор, то вместо символа $c_{\omega_{\infty}}$ для обозначения совокупности всех тотально $\omega$-композиционных формаций обычно используют символ $c_{\infty}^{\omega}$.

Пусть $\mathfrak{X}$ - некоторая совокупность групп. Через $c_{\omega_{\infty}}^{\tau}$ form $\mathfrak{X}$ обозначают пересечение всех $\tau$ замкнутых тотально $\omega$-композиционных формаций, содержащих $\mathfrak{X}$. Формацию $c_{\omega_{\infty}}^{\tau}$ form $\mathfrak{X}$ называют $\tau$-замкнутой тотально $\omega$-композиционной формацией, порожденной совокупностью групп $\mathfrak{X}$. Если $\mathfrak{X}=\{G\}$, то $c_{\omega_{\infty}}^{\tau}$ form $\mathfrak{X}=c_{\omega_{\infty}}^{\tau}$ form $G$ называют однопорожденной $\tau$-замкнутой тотально $\omega$-композиционной формачией.

Для любых $\tau$-замкнутых тотально $\omega$-композиционных формаций $\mathfrak{M}$ и $\mathfrak{H}$ полагают

$$
\mathfrak{M} \vee_{\omega_{\infty}}^{\tau^{c}} \mathfrak{H}=c_{\omega_{\infty}}^{\tau} \text { form }(\mathfrak{M} \cup \mathfrak{H})
$$

При этом для обозначения верхней грани в решетках $c_{\infty}^{\tau}$ и $c_{\infty}^{\omega}$ будем использовать символы $\mathrm{V}_{\infty}^{\tau^{c}}$ и $\vee_{\infty}^{\omega^{c}}$ соответственно.

Ввиду теоремы 1.6.4 [Воробьев, 2012, с. 70] множество всех $\tau$-замкнутых тотально $\omega$-композиционных формаций $c_{\omega_{\infty}}^{\tau}$, частично упорядоченное по включению, относительно операций $\cap$ и $\vee_{\omega_{\infty}}^{\tau^{c}}$ является полной решеткой формаций.

$\omega$-Композиционный спутник, все значения которого суть $c_{\omega_{\infty}}^{\tau}$-формации, называется $c_{\omega_{\infty}}^{\tau}$-значным.

Пусть $\left\{f_{i} \mid i \in I\right\}$ - некоторая система $c_{\omega_{\infty}}^{\tau}$-значных спутников. Тогда через $\vee_{\omega_{\infty}}^{\tau^{c}}\left(f_{i} \mid i \in I\right)$ обозначается такой спутник $f$, что $f(a)=c_{\omega_{\infty}}^{\tau}$ form $\left(\cup_{i \in I} f_{i}(a)\right)$ для всех $a \in \omega \cup\left\{\omega^{\prime}\right\}$, если по крайней мере одна из формаций $f_{i}(a) \neq \varnothing$. В противном случае полагают $f(a)=\varnothing$.

Пусть $\left\{f_{i} \mid i \in I\right\}$-набор всех $\omega$-композиционных $c_{\omega_{\infty}}^{\tau}$-значных спутников формации $\mathfrak{F}$. Тогда в силу леммы 2 [Скиба, Шеметков, 2000] $f=\bigcap_{i \in I} f_{i}-\omega$-композиционный $c_{\omega_{\infty}}^{\tau}$-значный спутник формации $\mathfrak{F}$, называемый минимальным.

В дальнейшем нам понадобятся некоторые известные факты теории формаций конечных групп, которые мы сформулируем в виде следующих лемм.

Лемма 1.1. [Скиба, Шеметков, 2000]. Пусть $\mathfrak{X}-$ непустая совокупность групn, $\mathfrak{F}=\Theta^{\omega_{c}}$ form $(\mathfrak{X})$, $\pi=\pi(\operatorname{Com}(\mathfrak{F})) \cap \omega$, и пусть $f$-минимальный $\omega$-композиционный $\Theta$-значный спутник формации F. Тогда справедливы следующие утверждения:

1) $f\left(\omega^{\prime}\right)=\Theta$ form $\left(G / R_{\omega}(G) \mid G \in \mathfrak{F}\right)$;

2) $f(p)=\Theta$ form $\left(\mathfrak{X}\left(C^{p}\right)\right)$ для всех $p \in \pi$;

3) $f(p)=\varnothing \partial л я$ всех $p \in \omega \backslash \pi$;

4) если $\mathfrak{F}=C F_{\omega}(h)$ и спутник $h \Theta$-значен, то для всех $p \in \pi$ имеет место

$$
f(p)=\Theta \operatorname{form}\left(G \mid G \in h(p) \cap \mathfrak{F}, O_{p}(G)=1\right)
$$

$u$

$$
f\left(\omega^{\prime}\right)=\Theta \text { form }\left(G \mid G \in h\left(\omega^{\prime}\right) \cap \mathfrak{F}, R_{\omega}(G)=1\right) ;
$$

5) если $E(\mathcal{K}(\mathfrak{X})) \in \Theta^{\omega_{c}}$, mо $\mathcal{K}(\mathfrak{X})=\mathcal{K}(\mathfrak{F})$.

Лемма 1.2. [Скиба, Шеметков, 2000]. Пусть формация $\mathfrak{F}=\mathfrak{M} \mathfrak{H}$, әде $\mathfrak{H}=C F_{\omega}(h), \mathfrak{M}=C F_{\omega}(m)$ и спутники $h$ и т являются внутренними. Тогда если $\mathfrak{N}_{\pi(\mathfrak{M})} \subseteq \mathfrak{M}$, то $\mathfrak{F}=C F_{\omega}(f)$, где $f\left(\omega^{\prime}\right)=\mathfrak{F}$ $u$

$$
f(p)= \begin{cases}m(p) \mathfrak{H}, & \text { если } p \in \pi(\operatorname{Com}(\mathfrak{M})) \cap \omega, \\ h(p), & \text { если } p \in \omega \backslash \pi(\operatorname{Com}(\mathfrak{M})) .\end{cases}
$$


Лемма 1.3. [Воробьев, 2012, с. 66]. Пусть $\mathfrak{F - р а з р е ш и м о ~ \omega - н а с ы щ е н н н а я ~ ф о р м а ц и я . ~ Т о г д а ~ с п р а - ~}$ ведливы следующие утверждения:

1) если $\mathfrak{F}$ имеет $\tau$-значный $\omega$-композиционный спутник, то $\mathfrak{F}-\tau$-замкнутая формация;

2) если $\mathfrak{F}-\tau$-замкнутая формация, то ее канонический $\omega$-композиционный спутник является $\tau$-значным.

Лемма 1.4. [Скиба, Шеметков, 2000]. Пусть $\Theta-$ такая полная решетка формаций, что для любой формачии $\mathfrak{H} \in \Theta$ бормачия $\mathfrak{N}_{p} \mathfrak{H} \in \Theta$ при любом $p \in \omega, \Theta^{\omega_{c}} \subseteq \Theta$. Тогда если $\mathfrak{F}=C F_{\omega}(F) \in$ $\Theta^{\omega_{c}}$, то спутник $F \Theta-$ значен.

Лемма 1.5. [Сафонов, 2007]. Пусть $\mathfrak{M}-$ непустая наследственная формачия, $\mathfrak{F}-$ непустая $\tau$ замкнутая формация. Тогда $\mathfrak{M F}-\tau$-замкнутая формация.

Лемма 1.6. [Скиба, 1997, с. 41]. Пусть А-монолитическая группа с неабелевым монолитом, $\mathfrak{M - ~ н е к о т о р а я ~} \tau$-замкнутая полуформация $u A \in l_{n}^{\tau}$ form $\mathfrak{M}$. Тогда $A \in \mathfrak{M}$.

Лемма 1.7. [Скиба, 1997, с. 179]. Решетка $l_{n}^{\tau}$ является алгебраической.

Лемма 1.8. [Скиба, Шеметков, 2000]. Если $\mathfrak{F}=C F_{\omega}(f) u$

$$
G / O_{p}(G) \in \mathfrak{F} \cap f(p)
$$

для некоторого $p \in \omega$, mо $G \in \mathfrak{F}$.

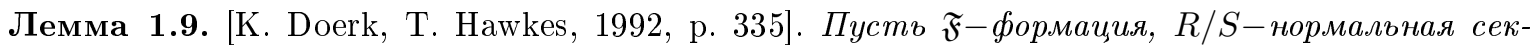
ция $\mathfrak{F}$-группы $G$ и $K$-нормальная подгруппа группы $G$, содержащаяся в $C_{G}(R / S)$. Относительно следующего действия группы $G / K$ на $R / S$ :

$$
(r S)^{g K}=g^{-1} r g S, \quad r \in R, g \in G,
$$

составим полупрямое произведение $H=(R / S) \lambda(G / K)$. Тогда $H \in \mathfrak{F}$.

2. Индуктивность решетки $c_{\omega_{\infty}}^{\tau}$ всех $\tau$-замкнутых тотально $\omega$-композиционных формаций. Целью данного раздела является доказательство индуктивности широкого класса полных решеток частично композиционных формаций при достаточно общих предположениях (теорема 2.1.). В качестве основного следствия мы получим индуктивность решетки $c_{\omega_{\infty}}^{\tau}$ (теорема 2.2.).

Для доказательства теорем установим несколько вспомогательных утверждений.

Лемма 2.1. Решетка $c_{\omega_{\infty}}^{\tau}$ всех $\tau$-замкнутых тоталъно $\omega$-композиционных формаций является частичной алгеброй формаций.

Доказательство. Проверим, что для любого простого числа $p$ и всякой $\tau$-замкнутой тотально разрешимо $\omega$-насыщенной формации $\mathfrak{H}$ формация $\mathfrak{M}=\mathfrak{N}_{p} \mathfrak{H}$ также является $\tau$-замкнутой тотально разрешимо $\omega$-насыщенной.

Поскольку формация $\mathfrak{H}-\tau$-замкнутая, то по лемме 1.5 . формация $\mathfrak{M}=\mathfrak{N}_{p} \mathfrak{H}$ также является $\tau$-замкнутой формацией.

Докажем, что формация $\mathfrak{M}$ тотально разрешимо $\omega$-насыщена. Обозначим через $h$ внутренний $\omega$-композиционный $c_{\omega_{\infty}}^{\tau}-$ значный спутник формации $\mathfrak{H}$.

Пусть вначале $p \in \omega$. Формация $\mathfrak{N}_{p}$ имеет такой внутренний $\omega$-композиционный спутник $m$, что $m(p)=(1), m\left(\omega^{\prime}\right)=(1)$ и $m(q)=\varnothing$ для всех $q \in \omega \backslash\{p\}$. Ввиду леммы 1.2. формация $\mathfrak{M}$ имеет спутник $f$, удовлетворяющий условиям: $f(p)=\mathfrak{H}, f\left(\omega^{\prime}\right)=\mathfrak{M}$ и $f(q)=h(q)$ для любого $q \in \omega \backslash\{p\}$. Поскольку $\mathfrak{H} \in c_{\omega_{\infty}}^{\tau}$, то $\mathfrak{M}$ является $n$-кратно разрешимо $\omega$-насыщенной для любого натурального $n$. Следовательно, $\mathfrak{M - т о т а л ь н о ~ р а з р е ш и м о ~} \omega$-насыщенная формация.

Пусть теперь $p \notin \omega$. В этом случае формация $\mathfrak{N}_{p}$ имеет такой внутренний $\omega$-композиционный спутник $m$, что $m(q)=\varnothing$ для всех $q \in \omega$ и $m\left(\omega^{\prime}\right)=\mathfrak{N}_{p}$. Тогда согласно лемме 1.2., формация $\mathfrak{M}$ имеет спутник $f$, такой, что $f(q)=h(q)$ для любого $q \in \omega$ и $f\left(\omega^{\prime}\right)=\mathfrak{M}$. Следовательно, М-тотально разрешимо $\omega$-насыщенная формация. Таким образом, $\mathfrak{M} \in c_{\omega_{\infty}}^{\tau}$.

Лемма 2.2. Пусть $\mathfrak{F}=C F_{\omega}(F)-\tau$-замкнутая тотально $\omega$-композиционная формация. Тогда канонический спутник $F$ является $c_{\omega_{\infty}}^{\tau}$-значным.

Доказательство. Прежде всего заметим, что $F\left(\omega^{\prime}\right)=\mathfrak{F} \in c_{\omega_{\infty}}^{\tau}$. Кроме того, из леммы 1.1 следует, что если $p \in \omega \backslash \pi(\operatorname{Com}(\mathfrak{F}))$, то $F(p)=\varnothing \in c_{\omega_{\infty}}^{\tau}$. Покажем, что для любого $p \in \pi(\operatorname{Com}(\mathfrak{F})) \cap \omega$ имеет место $F(p) \in c_{\omega_{\infty}}^{\tau}$. Поскольку $\mathfrak{F} \in c_{\infty}^{\omega}$, то $\mathfrak{F}=C F_{\omega}(f)$, где спутник $f$ является $c_{\infty}^{\omega}$-значным. Поэтому $\mathfrak{F} \in\left(c_{\infty}^{\omega}\right)^{\omega_{c}}$. Тогда, учитывая очевидное включение $\left(c_{\infty}^{\omega}\right)^{\omega_{c}} \subseteq c_{\infty}^{\omega}$ и лемму 2.1, из леммы 1.4 получаем $F(p) \in c_{\infty}^{\omega}$. Из первого утверждения леммы 1.3 следует, что формация $F(p)$ является $\tau$ замкнутой. Таким образом, формация $F(a) \in c_{\omega_{\infty}}^{\tau}$ для всех $a \in \omega \cup\left\{\omega^{\prime}\right\}$. Следовательно, спутник $F$ является $c_{\omega_{\infty}}^{\tau}$-значным.

Непосредственно из определений и первого утверждения леммы 1.3 вытекает следующая лемма.

Лемма 2.3. Пусть $\mathfrak{F}=C F_{\omega}(f)$, где $f-\omega$-композиционный $c_{\omega_{\infty}}^{\tau}$-значный спутник формации $\mathfrak{F}$. Тогда $\mathfrak{F}$ является $\tau$-замкнутой тотально $\omega$-композиционной формацией.

Следующий результат является прямым следствием лемм 2.2 и 2.3. 
Лемма 2.4. Справедливо равенство $\left(c_{\omega_{\infty}}^{\tau}\right)^{\omega_{c}}=c_{\omega_{\infty}}^{\tau}$.

В дальнейшем, учитывая замечание 3 [Скиба, Шеметков, 2000], мы полагаем $\mathfrak{L}=\mathfrak{L}^{+}$, при этом $\pi(\mathfrak{L})=\omega$. Учитывая также, что для любой полной решетки формаций $\Theta$ совокупность $\Theta^{\omega_{c}}$ всех формаций, которые имеют $\omega$-композиционный $\Theta$-значный спутник, - полная решетка формаций (см. подробнее [Скиба, Шеметков, 2000]), обозначим через $\mathfrak{M}$ такую формацию из $\Theta^{\omega_{c}}$, что $\mathfrak{H} \subseteq \mathfrak{M}$ для всех $\mathfrak{H} \in \Theta^{\omega_{c}}$.

Лемма 2.5. Пусть $\Theta-$ полная решетка формаций, $\mathfrak{X}-$ такая непустая совокупность групп, что $(\mathfrak{X}) \subseteq \mathfrak{M}, \mathfrak{F}=\Theta^{\omega_{c}}$ form $(\mathfrak{X})$. Предположим также, что существуют такие классы простых групп $\mathfrak{T}$ и $\mathfrak{L}_{1}$, что $\mathcal{K}(\mathfrak{X}) \subseteq \mathfrak{T} \subseteq \mathcal{K}(\mathfrak{M}), \mathfrak{T} \cap \mathfrak{L}_{1}=\mathcal{K}(\mathfrak{X}) \cap \mathfrak{L}_{1}$, причем $\mathfrak{L} \subseteq \mathfrak{L}_{1}$. Тогда если $E(\mathfrak{T}) \in \Theta^{\omega_{c}}$, то

$$
\mathcal{K}(\mathfrak{X}) \cap \mathfrak{L}_{1}=\mathcal{K}(\mathfrak{F}) \cap \mathfrak{L}_{1} .
$$

В частности,

$$
\pi(\operatorname{Com}(\mathfrak{X})) \cap \omega=\pi(\operatorname{Com}(\mathfrak{F})) \cap \omega .
$$

Доказательство. Поскольку $\mathcal{K}(\mathfrak{X}) \subseteq \mathfrak{T}$ и $E(\mathfrak{T}) \in \Theta^{\omega_{c}}$, то, учитывая равенсто $\mathcal{K}(E(\mathfrak{T}))=\mathfrak{T}$, имеем $(\mathfrak{X}) \subseteq E(\mathfrak{T})$. Поэтому $\mathfrak{F}=\Theta^{\omega_{c}}$ form $(\mathfrak{X}) \subseteq E(\mathfrak{T})$. Из последнего включения в силу равенства $\mathfrak{T} \cap \mathfrak{L}_{1}=\mathcal{K}(\mathfrak{X}) \cap \mathfrak{L}_{1}$ получаем

$$
\mathcal{K}(\mathfrak{F}) \cap \mathfrak{L}_{1} \subseteq \mathfrak{T} \cap \mathfrak{L}_{1}=\mathcal{K}(\mathfrak{X}) \cap \mathfrak{L}_{1} .
$$

Из включения $\mathfrak{X} \subseteq \mathfrak{F}$ следует, что $\mathcal{K}(\mathfrak{X}) \cap \mathfrak{L}_{1} \subseteq \mathcal{K}(\mathfrak{F}) \cap \mathfrak{L}_{1}$. Следовательно,

$$
\mathcal{K}(\mathfrak{X}) \cap \mathfrak{L}_{1}=\mathcal{K}(\mathfrak{F}) \cap \mathfrak{L}_{1} .
$$

В частности, полагая $\mathfrak{L}_{1}=\mathfrak{L}$ и учитывая, что класс $\mathfrak{L}$ содержит только абелевы простые группы (порядки которых принадлежат $\omega$ ), из последнего равенства имеем

$$
\operatorname{Com}(\mathfrak{X}) \cap \mathfrak{L}=\operatorname{Com}(\mathfrak{F}) \cap \mathfrak{L} .
$$

Следовательно,

$$
\pi(\operatorname{Com}(\mathfrak{X})) \cap \omega=\pi(\operatorname{Com}(\mathfrak{F})) \cap \omega,
$$

что и завершает доказательство леммы.

Лемма 2.6. Пусть $\mathfrak{T}$-непустой класс простых групп, $\mathfrak{R}=E(\mathfrak{T})$, и пусть $r$-такой $\omega$-композиционный спутник, что $r\left(\omega^{\prime}\right)=\mathfrak{R}$ и

$$
r(p)= \begin{cases}\mathfrak{R}, & \text { если } p \in \pi(\operatorname{Com}(\mathfrak{T})) \cap \omega, \\ \varnothing, & \text { если } p \in \omega \backslash \pi(\operatorname{Com}(\mathfrak{T})) .\end{cases}
$$

Тогда $\mathfrak{R}=C F_{\omega}(r)-\tau$-замкнутая тотально $\omega$-композиционная формация, а $r=R$ - канонический $c_{\omega_{\infty}}^{\tau}$-значный спутник.

Доказательство. Заметим, что $\mathcal{K}(\mathfrak{R})=\mathcal{K}(E(\mathfrak{T}))=\mathfrak{T}$. Поэтому $\operatorname{Com}(\mathfrak{R})=\operatorname{Com}(\mathfrak{T})$. Пусть $\pi=\pi(\operatorname{Com}(\mathfrak{T})) \cap \omega$.

Покажем, что $\mathfrak{R}=C F_{\omega}(r)$. Включение $\mathfrak{R} \subseteq C F_{\omega}(r)$ очевидно. Предположим, что обратное включение неверно и $G$ - группа минимального порядка из $C F_{\omega}(r) \backslash \mathfrak{R}$ с монолитом $P=G^{\Re}$.

Если $\pi(\operatorname{Com}(P)) \cap \omega=\varnothing$, то $R_{\omega}(G)=1$. Значит,

$$
G \cong G / 1=G / R_{\omega}(G) \in r\left(\omega^{\prime}\right)=\mathfrak{R} .
$$

Противоречие.

Следовательно, $\pi(\operatorname{Com}(P)) \cap \omega \neq \varnothing$. Пусть $p \in \pi(\operatorname{Com}(P)) \cap \omega$. Тогда $P$ - абелева $p$-группа. Если $p \notin \pi$, то ввиду условий $r(p)=\varnothing$ и $p \in \omega$, имеем $p \notin \pi(\operatorname{Com}(G)) \cap \omega$. Учитывая, что $p \in \omega$, получаем $p \notin \pi(\operatorname{Com}(G))$. Противоречие. Значит, $p \in \pi$. Следовательно,

$$
G \in \mathfrak{N}_{p} \mathfrak{R}=\mathfrak{N}_{p} E(\mathfrak{T})=E(\mathfrak{T})=\mathfrak{R} .
$$

Вновь полученное противоречие показывает, что $C F_{\omega}(r) \subseteq \mathfrak{R}$. Таким образом, $\mathfrak{R}=C F_{\omega}(r)$. $\mathrm{B}$ таком случае формация $\mathfrak{R}$ является $n$-кратно $\omega$-композиционной для любого натурального $n$. Следовательно, $\mathfrak{R}-$ тотально $\omega$-композиционна.

Кроме того, несложно видеть, что $\mathfrak{R}=E(\mathfrak{T})$ является $\mathrm{S}_{n}$-замкнутой формацией. Поэтому, ввиду ограничения на подгрупповой функтор $\tau$ (для любой группы $G$ все подгруппы, входящие в $\tau(G)$, субнормальны в $G)$, окончательно заключаем, что $\mathfrak{R}$ является $\tau$-замкнутой тотально $\omega$-композиционной формацией.

Поскольку для любого $p \in \pi$ имеет место $\mathfrak{N}_{p} \mathfrak{R}=\mathfrak{N}_{p} E(\mathfrak{T})=E(\mathfrak{T})=\mathfrak{R}$, заключаем, что $r$ является каноническим $c_{\omega_{\infty}}^{\tau}$-значным спутником формации $\mathfrak{R}$ (см. также лемма 2.2$)$. 


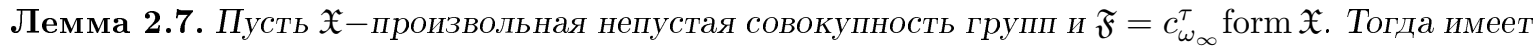
место равенство

$$
\operatorname{Com}(\mathfrak{X})=\operatorname{Com}(\mathfrak{F}) .
$$

В частности,

$$
\pi(\operatorname{Com}(\mathfrak{X})) \cap \omega=\pi(\operatorname{Com}(\mathfrak{F})) \cap \omega .
$$

Доказательство. Обозначим через $\mathfrak{I}^{+}$класс всех простых абелевых групп, а через $\mathfrak{I}^{-}-$класс всех простых неабелевых групп $\left(\mathfrak{I}^{-}=\mathfrak{I}^{\prime}=\mathfrak{I} \backslash \mathfrak{I}^{+}\right)$. Положим $\mathfrak{T}=\operatorname{Com}(\mathfrak{X}) \cup \mathfrak{I}^{-}, \mathfrak{L}_{1}=\mathfrak{I}^{+}, \mathfrak{M}=\mathfrak{G}$ $\left(\mathfrak{G}-\right.$ класс всех групп). Покажем, что для решетки $\Theta=c_{\omega_{\infty}}^{\tau}$ выполнены условия леммы 2.5.

Заметим сначала, что согласно теореме 1.6.4 [Воробьев, 2012, с. 70] $\mathfrak{M}=\mathfrak{G}$ является наибольшим элементом решетки $\Theta=c_{\omega_{\infty}}^{\tau}$. Далее, несложно видеть, что ввиду выбора классов $\mathfrak{T}$ и $\mathfrak{L}_{1}$ имеют место включения $\mathcal{K}(\mathfrak{X}) \subseteq \mathfrak{T} \subseteq \mathcal{K}(\mathfrak{M})=\mathfrak{I}, \mathfrak{L} \subseteq \mathfrak{L}_{1}$. Кроме того, так как $\mathfrak{L}_{1}=\mathfrak{I}^{+}-$класс всех простых абелевых групп, то имеют место равенства

$$
\mathfrak{T} \cap \mathfrak{L}_{1}=\operatorname{Com}(\mathfrak{T})=\operatorname{Com}(\mathfrak{X})=\mathcal{K}(\mathfrak{X}) \cap \mathfrak{L}_{1} .
$$

Наконец, из леммы 2.6 следует, что $E(\mathfrak{T}) \in c_{\omega_{\infty}}^{\tau}$.

Таким образом, все условия леммы 2.5 выполнены. Применяя ее с учетом равенства $\mathfrak{L}_{1}=\mathfrak{I}^{+}$, получаем

$$
\operatorname{Com}(\mathfrak{X})=\mathcal{K}(\mathfrak{X}) \cap \mathfrak{L}_{1}=\mathcal{K}(\mathfrak{F}) \cap \mathfrak{L}_{1}=\operatorname{Com}(\mathfrak{F}) .
$$

Последнее утверждение леммы непосредственно следует из уже доказанного.

Ввиду лемм 2.4 и 2.7 из леммы 1.1 вытекает следующий результат.

Лемма 2.8. Пусть $\mathfrak{X}-$ непустая совокупность групп, $\mathfrak{F}=c_{\omega_{\infty}}^{\tau}$ form $\mathfrak{X}, f-$ минимальный $\omega$-композиционный $c_{\omega_{\infty}}^{\tau}$-значный спутник формации $\mathfrak{F}$, и пусть $\pi=\pi(\operatorname{Com}(\mathfrak{X})) \cap \omega$. Тогда справедливы следующие утверждения:

1) $f\left(\omega^{\prime}\right)=c_{\omega_{\infty}}^{\tau}$ form $\left(G / R_{\omega}(G) \mid G \in \mathfrak{X}\right)$;

2) $f(p)=c_{\omega_{\infty}}^{\tau}$ form $\left(G / C^{p}(G) \mid G \in \mathfrak{X}\right)$ для всех $p \in \pi$;

3) $f(p)=\varnothing$ для всех $p \in \omega \backslash \pi$;

4) если $\mathfrak{F}=C F_{\omega}(h)$ и спутник $h c_{\omega_{\infty}}^{\tau}$-значен, то для всех $p \in \pi$ имеет место

$$
f(p)=c_{\omega_{\infty}}^{\tau} \text { form }\left(G \mid G \in h(p) \cap \mathfrak{F}, O_{p}(G)=1\right)
$$

и

5) $\pi(\operatorname{Com}(\mathfrak{X})) \cap \omega=\pi(\operatorname{Com}(\mathfrak{F})) \cap \omega$.

Лемма 2.9. Пусть $\Theta-$ такая полная решетка формаций, что для любой непустой совокупности групп $\mathfrak{X}$, такой, что $(\mathfrak{X}) \subseteq \mathfrak{M}$, имеет место $\pi(\operatorname{Com}(\mathfrak{X})) \cap \omega=\pi(\operatorname{Com}(\mathfrak{F})) \cap \omega$, где $\mathfrak{F}=\Theta^{\omega_{c}}$ form $(\mathfrak{X}) . И$ пусть $f_{i}-$ минимальный $\omega$-композиционный $\Theta$-значный спутник формации $\mathfrak{F}_{i} \in \Theta^{\omega_{c}}$, где $i \in I$. Тогда $\vee_{\Theta}\left(f_{i} \mid i \in I\right)$ - минимальный $\omega$-композиционный $\Theta$-значный спутник формации $\mathfrak{F}=\vee_{\Theta \omega_{c}}\left(\mathfrak{F}_{i} \mid i \in I\right)$.

Доказательство. Пусть $f=\vee_{\Theta}\left(f_{i} \mid i \in I\right)$ и $h$ - минимальный $\omega$-композиционный $\Theta$-значный спутник формации $\mathfrak{F}$. Так как $\mathfrak{F}_{i} \subseteq \mathfrak{M}$ для любого $i \in I$, то $\underset{i \in I}{\cup} \mathfrak{F}_{i} \subseteq \mathfrak{M}$. Следовательно, ввиду условия леммы, имеем $\pi\left(\operatorname{Com}\left(\cup_{i \in I} \mathfrak{F}_{i}\right)\right) \cap \omega=\pi(\operatorname{Com}(\mathfrak{F})) \cap \omega$. Пусть $\pi=\pi\left(\operatorname{Com}\left(\underset{i \in I}{\cup} \mathfrak{F}_{i}\right)\right) \cap \omega$.

Покажем, что $h=f$. Если $p \in \omega \backslash \pi$, то согласно лемме 1.1 для любого $i \in I$ имеет место $f_{i}(p)=\varnothing$. Значит, $f(p)=\varnothing$. Понятно также, что $h(p)=\varnothing$.

Пусть теперь $p \in \pi \cap \omega$. Тогда найдется такое $i \in I$, что $f_{i}(p) \neq \varnothing$. Ввиду леммы 1.1 имеют место равенства

$$
\begin{gathered}
h(p)=\Theta \text { form }\left(G / C^{p}(G) \mid G \in \cup_{i \in I}^{\cup} \mathfrak{F}_{i}\right)=\Theta \text { form }\left(\cup_{i \in I} \Theta \text { form }\left(G / C^{p}(G) \mid G \in \mathfrak{F}_{i}\right)\right)= \\
=\Theta \text { form }\left(\cup_{i \in I} f_{i}(p)\right)=\left(\vee_{\Theta}\left(f_{i} \mid i \in I\right)\right)(p)=f(p) .
\end{gathered}
$$

Кроме того, из леммы 1.1 следует, что

$$
\begin{aligned}
& h\left(\omega^{\prime}\right)=\Theta \text { form }\left(G / R_{\omega}(G) \mid G \in \underset{i \in I}{\cup} \mathfrak{F}_{i}\right)=\Theta \text { form }\left(\cup_{i \in I} \Theta \text { form }\left(G / R_{\omega}(G) \mid G \in \mathfrak{F}_{i}\right)\right)= \\
&=\Theta \text { form }\left(\cup_{i \in I} f_{i}\left(\omega^{\prime}\right)\right)=\left(\vee_{\Theta}\left(f_{i} \mid i \in I\right)\right)\left(\omega^{\prime}\right)=f\left(\omega^{\prime}\right) .
\end{aligned}
$$

Таким образом, $h=f$.

Полагая в лемме $2.9 \Theta=c_{\omega_{\infty}}^{\tau}$ и учитывая лемму 2.7 , получаем следующую лемму. 
Лемма 2.10. Пусть $f_{i}-$ минимальный $\omega$-композиционный $c_{\omega_{\infty}}^{\tau}-$ значный спутник $\tau$-замкнутой тотально $\omega$-композиционной формации $\mathfrak{F}_{i}$, где $i \in I$. Тогда $\vee_{\omega_{\infty}}^{\tau^{c}}\left(f_{i} \mid i \in I\right)-$ минимальный $\omega-$ композиционный $c_{\omega_{\infty}}^{\tau}$-значный спутник формации $\mathfrak{F}=\vee_{\omega_{\infty}}^{\tau^{c}}\left(\mathfrak{F}_{i} \mid i \in I\right)$.

Теперь мы готовы доказать основной результат данного раздела.

Теорема 2.1. Пусть $\Theta$ - такая полная решетка формаций, что для любой непустой совокупности групп $\mathfrak{X}$, такой, что $(\mathfrak{X}) \subseteq \mathfrak{M}$, имеет место $\pi(\operatorname{Com}(\mathfrak{X})) \cap \omega=\pi(\operatorname{Com}(\mathfrak{F})) \cap \omega$, где $\mathfrak{F}=\Theta^{\omega_{c}}$ form $(\mathfrak{X})$. И пусть для любой формации $\mathfrak{H} \in \Theta$ формация $\mathfrak{N}_{p} \mathfrak{H} \in \Theta$ для всех $p \in \omega, \Theta^{\omega_{c}} \subseteq \Theta$. Тогда решетка формаций $\Theta$ индуктивна.

Доказательство. Пусть $\left\{\mathfrak{F}_{i}=C F_{\omega}\left(f_{i}\right) \mid i \in I\right\}$ - произвольный набор формаций из $\Theta^{\omega_{c}}$, где $f_{i}-$ некоторый внутренний $\omega$-композиционный $\Theta$-значный спутник. Обозначим через $\mathfrak{F}$ формацию $\vee_{\Theta \omega_{c}}\left(\mathfrak{F}_{i} \mid i \in I\right)$. Покажем, что $\mathfrak{F}=C F_{\omega}\left(\vee_{\Theta}\left(f_{i} \mid i \in I\right)\right)$.

Введем следующие обозначения: $h_{i}-$ минимальный $\omega$-композиционный $\Theta$-значный спутник формации $\mathfrak{F}_{i}=C F_{\omega}\left(F_{i}\right), i \in I, \mathfrak{F}=C F_{\omega}(F), h$-минимальный $\omega$-композиционный $\Theta$-значный спутник формации $\mathfrak{F}$. Кроме того, считаем, что $p \in \omega$. Тогда согласно лемме $2.9 h=\vee_{\Theta}\left(h_{i} \mid i \in I\right)$. Ввиду замечания 1 [Скиба, Шеметков, 2000] и леммы 1.4 имеют место включения

$$
h_{i}(p) \subseteq f_{i}(p) \subseteq \mathfrak{N}_{p} h_{i}(p)=F_{i}(p) \in \Theta
$$

Ввиду того, что

$$
\mathfrak{N}_{p} h_{i}(p) \subseteq \mathfrak{N}_{p}\left(\vee_{\Theta}\left(h_{i}(p) \mid i \in I\right)\right) \text { и } \mathfrak{N}_{p}\left(\vee_{\Theta}\left(h_{i}(p) \mid i \in I\right)\right) \in \Theta
$$

имеем

$$
\Theta \text { form }\left(\cup_{i \in I}^{\cup} \mathfrak{N}_{p} h_{i}(p)\right) \subseteq \Theta \text { form }\left(\mathfrak{N}_{p}\left(\vee_{\Theta}\left(h_{i}(p) \mid i \in I\right)\right)\right)=\mathfrak{N}_{p}\left(\vee_{\Theta}\left(h_{i}(p) \mid i \in I\right)\right)
$$

Следовательно,

$$
\begin{gathered}
h(p)=\Theta \text { form }\left(\cup_{i \in I} h_{i}(p)\right) \subseteq \Theta \text { form }\left(\cup_{i \in I} f_{i}(p)\right)=f(p) \subseteq \Theta \text { form }\left(\cup_{i \in I} \mathfrak{N}_{p} h_{i}(p)\right) \subseteq \\
\subseteq \mathfrak{N}_{p}\left(\vee_{\Theta}\left(h_{i}(p) \mid i \in I\right)\right)=\mathfrak{N}_{p} h(p)=F(p) .
\end{gathered}
$$

Итак, $h(p) \subseteq f(p) \subseteq F(p)$ для всех $p \in \omega$.

Кроме того, поскольку

$$
h_{i}\left(\omega^{\prime}\right) \subseteq f_{i}\left(\omega^{\prime}\right) \subseteq F_{i}\left(\omega^{\prime}\right)=\mathfrak{F}_{i} \in \Theta^{\omega_{c}} \text { и } \Theta^{\omega_{c}} \subseteq \Theta,
$$

справедливы включения

$$
\begin{gathered}
h\left(\omega^{\prime}\right)=\Theta \text { form }\left(\cup_{i \in I} h_{i}\left(\omega^{\prime}\right)\right) \subseteq \Theta \text { form }\left(\cup_{i \in I} f_{i}\left(\omega^{\prime}\right)\right)=f\left(\omega^{\prime}\right) \subseteq \Theta \text { form }\left(\cup_{i \in I} F_{i}\left(\omega^{\prime}\right)\right)= \\
=\Theta \text { form }\left(\cup_{i \in I} \mathfrak{F}_{i}\right) \subseteq \Theta^{\omega_{c}} \text { form }\left(\cup_{i \in I} \mathfrak{F}_{i}\right)=\mathfrak{F}=F\left(\omega^{\prime}\right) .
\end{gathered}
$$

Значит, $h\left(\omega^{\prime}\right) \subseteq f\left(\omega^{\prime}\right) \subseteq F\left(\omega^{\prime}\right)$. Таким образом, $h \leq f \leq F$, и поэтому $\mathfrak{F}=C F_{\omega}(f)$.

Отметим, что доказанная теорема является обобщением соответствующего утверждения теоремы работы Н. Н. Воробьева [2000] на случай решеток частично композиционных формаций.

Из теоремы 2.1 ввиду доказательства леммы 4.6.1 [Воробьев, 2012, с. 213], а также лемм 4.6.3 и 4.6.4 [Воробьев, 2012, с. 216], вытекает

Следствие 2.1. [Воробьев, Царев, 2010; Жизневский, 2010]. Решетка $c_{\omega_{n}}^{\tau}$ всех $\tau$-замкнутых $n$-кратно $\omega$-композиционных формаций индуктивна.

Учитывая леммы 2.1, 2.4 и 2.7, из теоремы 2.1 получаем следующий результат, который является положительным ответом на вопрос 5.5(2) [Tsarev, Vorob'ev, 2018].

Теорема 2.2. Решетка $c_{\omega_{\infty}}^{\tau}$ всех $\tau$-замкнутых тотально $\omega$-композиционных формаций индуктивна.

Поскольку каждая полная подрешетка индуктивной решетки также является индуктивной (см. [Скиба, 1997, с. 155]), из теоремы 2.2 получаем

Следствие 2.2 Каждая полная подрешетка решетки $c_{\omega_{\infty}}^{\tau}$ индуктивна.

Отметим также следующие важные следствия.

Если $\tau$-тривиальный подгрупповой функтор, то из теоремы 2.2 вытекает

Следствие 2.3. Решетка $c_{\infty}^{\omega}$ всех тотально $\omega$-композиционных формаций индуктивна.

В случае, когда $\tau(G)=\mathrm{S}_{n}(G)$ для любой группы $G$, из теоремы 2.2 получаем

Следствие 2.4. Решетка всех нормально наследственных тотально $\omega$-композиционных формаций индуктивна. 
Если $\omega=\{p\}$, то из теоремы 2.2 имеем

Следствие 2.5. Решетка $c_{p_{\infty}}^{\tau}$ всех $\tau$-замкнутых тотально $p$-композиционньх формаций индуктивна.

Если $\omega=\mathbb{P}$-множество всех простых чисел, из теоремы 2.2 получаем

Следствие 2.6. [Tsarev, 2018]. Решетка $c_{\infty}^{\tau}$ всех $\tau$-замкнутых тотально композиционных формаций индуктивна.

3. Алгебраичность решетки $c_{\omega_{\infty}}^{\tau}$ всех $\tau$-замкнутых тотально $\omega$-композиционных формаций. Для доказательства алгебрачности решетки $c_{\omega_{\infty}}^{\tau}$ нам понадобятся следующие вспомогательные утверждения.

Лемма 3.1. Пусть $\mathfrak{F}-$ непустая $\tau$-замкнутая формация, $\pi$-такое множество простых чисел, что $\pi(\operatorname{Com}(\mathfrak{F})) \cap \omega \subseteq \pi$. Тогда формация $\mathfrak{S}_{\pi} \mathfrak{F}$ является $\tau$-замкнутой тотально $\omega$-композиционной формацией.

Доказательство. Если $\pi=\varnothing$, то ввиду примера 1 [Скиба, Шеметков, 2000] $\mathfrak{S}_{\varnothing} \mathfrak{F}=(1) \mathfrak{F}=$ $\mathfrak{F}$-тотально $\omega$-композиционна, и утверждение леммы верно.

Предположим, что $\pi \neq \varnothing$. Покажем, что формация $\mathfrak{S}_{\pi} \mathfrak{F}$ является тотально разрешимо $\omega-$ насыщенной. Пусть $\mathfrak{T}=\left(Z_{p} \mid p \in \pi\right)$, где $Z_{p}$ - группа простого порядка $p$. Тогда, очевидно, $\mathfrak{S}_{\pi}=$ $E(\mathfrak{T})$. Заметим также, что $\pi(\operatorname{Com}(\mathfrak{T}))=\pi\left(\operatorname{Com}\left(\mathfrak{S}_{\pi}\right)\right)=\pi$. Тогда ввиду леммы $2.6 \mathfrak{S}_{\pi}=C F_{\omega}(K)$, где $K\left(\omega^{\prime}\right)=\mathfrak{S}_{\pi}, K(p)=\mathfrak{S}_{\pi}$ при любом $p \in \pi \cap \omega$ и $K(p)=\varnothing$ при $p \in \omega \backslash \pi$. Из условия следует, что

$$
\pi(\operatorname{Com}(\mathfrak{F})) \cap \omega \subseteq \pi=\pi\left(\operatorname{Com}\left(\mathfrak{S}_{\pi}\right)\right) .
$$

Учитывая последнее включение, а также лемму 1.2 , заключаем, что формация $\mathfrak{S}_{\pi} \mathfrak{F}$ имеет такой $\omega$-композиционный спутник $t$, что $t\left(\omega^{\prime}\right)=\mathfrak{S}_{\pi} \mathfrak{F}, t(p)=K(p) \mathfrak{F}=\mathfrak{S}_{\pi} \mathfrak{F}$ при любом $p \in \pi \cap \omega$ и $t(p)=\varnothing$ при $p \in \omega \backslash \pi$ (см. также лемма 4.5 [Guo et al., 2007] и лемма 3.3.8 [Селькин, 2011]). Поэтому $\mathfrak{S}_{\pi} \mathfrak{F}$ является $n$-кратно разрешимо $\omega$-насыщенной для любого натурального $n$. Следовательно, $\mathfrak{S}_{\pi} \mathfrak{F}$-тотально разрешимо $\omega$-насыщенная формация.

Поскольку формация $\mathfrak{S}_{\pi}$ наследственна, то ввиду леммы 1.5 формация $\mathfrak{S}_{\pi} \mathfrak{F}$ является $\tau$-замкнутой. Таким образом, $\mathfrak{S}_{\pi} \mathfrak{F}-\tau$-замкнутая тотально $\omega$-композиционная формация.

Лемма 3.1. Пусть $\mathfrak{H}=c_{\omega_{\infty}}^{\tau}$ form $\left(\underset{i \in I}{\cup} \mathfrak{F}_{i}\right)$, где $\mathfrak{F}_{i}-\tau$-замкнутая тотально $\omega$-композиционная формация $(i \in I), A \in \mathfrak{H}-$ монолитическая группа. Тогда если $\operatorname{Soc}(A)$-неабелева группа, то $A \in \underset{i \in I}{\cup} \mathfrak{F}_{i}$.

Доказательство. Пусть $A$-группа из условия леммы, $\pi=\pi\left(\operatorname{Com}\left(\underset{i \in I}{\cup} \mathfrak{F}_{i}\right)\right) \cap \omega$. Ввиду леммы 2.7

$$
\pi\left(\operatorname{Com}\left(\cup_{i \in I} \mathfrak{F}_{i}\right)\right) \cap \omega=\pi(\operatorname{Com}(\mathfrak{H})) \cap \omega
$$

Значит, $\pi=\pi(\operatorname{Com}(\mathfrak{H})) \cap \omega$. Согласно лемме 3.1

$$
\mathfrak{S}_{\pi} \tau \text { form }\left(\cup_{i \in I} \mathfrak{F}_{i}\right) \in c_{\omega_{\infty}}^{\tau}
$$

Поэтому

$$
c_{\omega_{\infty}}^{\tau} \text { form }\left(\cup_{i \in I}^{\cup} \mathfrak{F}_{i}\right) \subseteq \mathfrak{S}_{\pi} \tau \text { form }\left(\cup_{i \in I} \mathfrak{F}_{i}\right) .
$$

Значит, $A \in \mathfrak{S}_{\pi} \tau$ form $\left(\cup_{i \in I} \mathfrak{F}_{i}\right)$. Поскольку $\operatorname{Soc}(A)$-неабелева группа, то

$$
A \in \tau \text { form }\left(\cup_{i \in I} \mathfrak{F}_{i}\right)
$$

Тогда вследствие леммы 1.6 заключаем, что $A \in \underset{i \in I}{\cup} \mathfrak{F}_{i}$.

Основным результатом работы является следующая теорема, которая дает положительный ответ на вопрос 5.5(1) [Tsarev, Vorob'ev, 2018].

Теорема 3.1. Решетка $c_{\omega_{\infty}}^{\tau}$ всех $\tau$-замкнутых тотально $\omega$-композиционных формаций является алгебраической.

Доказательство. Покажем вначале, что для любой группы $A$ однопорожденная $\tau$-замкнутая тотально $\omega$-композиционная формация $\mathfrak{F}=c_{\omega_{\infty}}^{\tau}$ form $A$ является компактным элементом решетки $c_{\omega_{\infty}}^{\tau}$

Предположим противное. Тогда существуют группа $A$ и формации $\mathfrak{F}_{i} \in c_{\omega_{\infty}}^{\tau}$, где $i \in I$, такие, что

и, кроме того,

$$
\mathfrak{F}=c_{\omega_{\infty}}^{\tau} \text { form } A \subseteq \mathfrak{H}=c_{\omega_{\infty}}^{\tau} \text { form }\left(\cup_{i \in I}^{\cup} \mathfrak{F}_{i}\right)
$$

$$
\mathfrak{F}=c_{\omega_{\infty}}^{\tau} \text { form } A \nsubseteq c_{\omega_{\infty}}^{\tau} \text { form }\left(\cup_{j \in J} \mathfrak{F}_{j}\right)
$$


для любого конечного подмножества $J \subset I$. Пусть $A$-группа минимального порядка среди групп с таким свойством. Покажем, что группа $A$ монолитична. Допустим, что $N_{1}$ и $N_{2}-$ две различные минимальные нормальные подгруппы группы $A$. Пусть $\mathfrak{L}=c_{\omega_{\infty}}^{\tau}$ form $\left(A / N_{1}\right), \mathfrak{M}=c_{\omega_{\infty}}^{\tau}$ form $\left(A / N_{2}\right)$.

Поскольку $\left|A / N_{1}\right|<|A|$ и $\left|A / N_{2}\right|<|A|$, то ввиду выбора группы $A$ из включений

$$
\begin{aligned}
& \mathfrak{L}=c_{\omega_{\infty}}^{\tau} \text { form } A / N_{1} \subseteq \mathfrak{H}=c_{\omega_{\infty}}^{\tau} \text { form }\left(\cup_{i \in I}^{\cup} \mathfrak{F}_{i}\right), \\
& \mathfrak{M}=c_{\omega_{\infty}}^{\tau} \text { form } A / N_{1} \subseteq \mathfrak{H}=c_{\omega_{\infty}}^{\tau} \text { form }\left(\cup_{i \in I} \mathfrak{F}_{i}\right)
\end{aligned}
$$

следует, что найдутся такие наборы индексов $i_{1}, \ldots, i_{k} \in I$ и $j_{1}, \ldots, j_{l} \in I$, что

$$
\begin{gathered}
\mathfrak{L} \subseteq c_{\omega_{\infty}}^{\tau} \text { form }\left(\mathfrak{F}_{i_{1}} \cup \ldots \cup \mathfrak{F}_{i_{k}}\right), \\
\mathfrak{M} \subseteq c_{\omega_{\infty}}^{\tau} \text { form }\left(\mathfrak{F}_{j_{1}} \cup \ldots \cup \mathfrak{F}_{j_{l}}\right)
\end{gathered}
$$

Следовательно,

$$
\mathfrak{F}=\mathfrak{L} \vee_{\omega_{\infty}}^{\tau^{c}} \mathfrak{M} \subseteq c_{\omega_{\infty}}^{\tau} \text { form }\left(\mathfrak{F}_{i_{1}} \cup \ldots \cup \mathfrak{F}_{i_{k}} \cup \mathfrak{F}_{j_{1}} \cup \ldots \cup \mathfrak{F}_{j_{l}}\right)
$$

Получили противоречие. Значит, $A$-монолитическая группа.

Пусть $P=\operatorname{Soc}(A)$. Предположим, что $P$-неабелева группа. Тогда, учитывая условие $A \in$ $c_{\omega_{\infty}}^{\tau}$ form $\left(\cup_{i \in I} \mathfrak{F}_{i}\right)$ и лемму 3.2 , имеем $A \in \cup_{i \in I} \mathfrak{F}_{i}$. Поэтому существует такой индекс $i_{0} \in I$, что $A \in \mathfrak{F}_{i_{0}}$. Учитывая, что $\mathfrak{F}_{i_{0}}-c_{\omega_{\infty}}^{\tau}$-формация, имеем $\mathfrak{F}=c_{\omega_{\infty}}^{\tau}$ form $A \subseteq \mathfrak{F}_{i_{0}}$. Противоречие.

Следовательно, $P$-абелева $p$-группа для некоторого простого числа $p$. Пусть

$$
\pi=\pi\left(\operatorname{Com}\left(\underset{i \in I}{\cup} \mathfrak{F}_{i}\right)\right) \cap \omega .
$$

Предположим, что $p \notin \omega$. Тогда $p \notin \pi$. Ввиду леммы $3.1 \mathfrak{S}_{\pi} \tau$ form $\left(\cup_{i \in I} \mathfrak{F}_{i}\right) \in c_{\omega_{\infty}}^{\tau}$. Значит,

$$
\mathfrak{H}=c_{\omega_{\infty}}^{\tau} \text { form }\left(\cup_{i \in I} \mathfrak{F}_{i}\right) \subseteq \mathfrak{S}_{\pi} \tau \text { form }\left(\cup_{i \in I} \mathfrak{F}_{i}\right) .
$$

Так как

$$
A \in \mathfrak{H}=c_{\omega_{\infty}}^{\tau} \text { form }\left(\cup_{i \in I} \mathfrak{F}_{i}\right)
$$

то имеет место

$$
A \in \mathfrak{S}_{\pi} \tau \text { form }\left(\cup_{i \in I} \mathfrak{F}_{i}\right) .
$$

Поскольку $p \notin \pi$, то

$$
A \in \tau \text { form }\left(\cup_{i \in I} \mathfrak{F}_{i}\right) .
$$

Тогда, согласно лемме 1.7, найдутся такие индексы $i_{1}, \ldots, i_{m} \in I$, что

$$
\tau \text { form } A \subseteq \tau \text { form }\left(\mathfrak{F}_{i_{1}} \cup \ldots \cup \mathfrak{F}_{i_{m}}\right) .
$$

Из включения

$$
\tau \text { form }\left(\mathfrak{F}_{i_{1}} \cup \ldots \cup \mathfrak{F}_{i_{m}}\right) \subseteq c_{\omega_{\infty}}^{\tau} \text { form }\left(\mathfrak{F}_{i_{1}} \cup \ldots \cup \mathfrak{F}_{i_{m}}\right),
$$

следует, что

Следовательно,

$$
\tau \text { form } A \subseteq c_{\omega_{\infty}}^{\tau} \text { form }\left(\mathfrak{F}_{i_{1}} \cup \ldots \cup \mathfrak{F}_{i_{m}}\right)
$$

$$
c_{\omega_{\infty}}^{\tau} \text { form } A \subseteq c_{\omega_{\infty}}^{\tau} \text { form }\left(\mathfrak{F}_{i_{1}} \cup \ldots \cup \mathfrak{F}_{i_{m}}\right) .
$$

Противоречие. Поэтому $p \in \omega$. Так как $A / P \in \mathfrak{H}$ и $|A / P|<|A|$, то в силу выбора группы $A$ существует такой набор индексов $i_{1}, \ldots, i_{s} \in I$, что

$$
A / P \in c_{\omega_{\infty}}^{\tau} \text { form }\left(\mathfrak{F}_{i_{1}} \cup \ldots \cup \mathfrak{F}_{i_{s}}\right) .
$$

Пусть $T=P \lambda\left(A / C_{A}(P)\right)$. Поскольку $A \in \mathfrak{F}$, то согласно лемме $1.9 T \in \mathfrak{F}$. Значит, $T \in \mathfrak{H}$.

Предположим, что $|T|<|A|$. Тогда ввиду выбора группы $A$ найдутся такие индексы $j_{1}, \ldots, j_{t} \in I$, что

$$
T \in c_{\omega_{\infty}}^{\tau} \text { form }\left(\mathfrak{F}_{j_{1}} \cup \ldots \cup \mathfrak{F}_{j_{t}}\right) .
$$

Пусть $\mathfrak{V}=c_{\omega_{\infty}}^{\tau}$ form $\left(\mathfrak{F}_{i_{1}} \cup \ldots \cup \mathfrak{F}_{i_{s}} \cup \mathfrak{F}_{j_{1}} \cup \ldots \cup \mathfrak{F}_{j_{t}}\right), v$ - некоторый $\omega$-композиционный спутник формации $\mathfrak{V}$.

Покажем, что в таком случае $A \in \mathfrak{V}$. Ясно, что $T \in \mathfrak{V}$ и $A / P \in \mathfrak{V}$. Из определения группы $T$ также следует, что

$$
A / C_{A}(P) \cong T / P=T / C_{T}(P)=T / C^{p}(T) \in v(p) .
$$


Пусть $C^{p}(A / P)=\widetilde{C} / P$. Поскольку $A / P \in \mathfrak{V}$, то имеет место

$$
A / \widetilde{C} \cong(A / P) /(\widetilde{C} / P)=(A / P) / C^{p}(A / P) \in v(p) .
$$

Учитывая, что $C^{p}(A)=\widetilde{C} \cap C_{A}(P)$, получаем

$$
A / C^{p}(A)=A /\left(\widetilde{C} \cap C_{A}(P)\right) \in v(p) .
$$

Кроме того, поскольку $P \subseteq R_{\omega}(A)$ и $P \subseteq C^{q}(A)$ для всех простых $q \neq p$, то

$$
R_{\omega}(A / P)=R_{\omega}(A) / P \text { и } C^{q}(A / P)=C^{q}(A) / P .
$$

Снова принимая во внимание, что $A / P \in \mathfrak{V}$, имеем

$$
A / R_{\omega}(A) \cong(A / P) /\left(R_{\omega}(A) / P\right)=(A / P) / R_{\omega}(A / P) \in v\left(\omega^{\prime}\right)
$$

и

$$
A / C^{q}(A) \cong(A / P) /\left(C^{q}(A) / P\right)=(A / P) / C^{q}(A / P) \in v(q)
$$

для любого $q \in(\pi(\operatorname{Com}(A)) \cap \omega) \backslash\{p\}$. Таким образом,

$$
A / C^{r}(A) \in v(r)
$$

для всех $\pi(\operatorname{Com}(A)) \cap \omega$. Следовательно, $A \in \mathfrak{V}$. В таком случае

$$
\mathfrak{F}=c_{\omega_{\infty}}^{\tau} \text { form } A \subseteq \mathfrak{V}=c_{\omega_{\infty}}^{\tau} \text { form }\left(\mathfrak{F}_{i_{1}} \cup \ldots \cup \mathfrak{F}_{i_{s}} \cup \mathfrak{F}_{j_{1}} \cup \ldots \cup \mathfrak{F}_{j_{t}}\right) .
$$

Противоречие.

Поэтому $|T|=|A|$. Следовательно, $P=C_{A}(P)$, что влечет

$$
P=C_{A}(P)=C^{p}(G)=F_{p}(A)=F(A)=O_{p}(A) .
$$

Пусть $f_{i}$ и $h$ - минимальные $\omega$-композиционные $c_{\omega_{\infty}}^{\tau}$-значные спутники формаций $\mathfrak{F}_{i}$ и $\mathfrak{H}$ соответственно $(i \in I)$. Тогда ввиду леммы 2.10

$$
h=\vee_{\omega_{\infty}}^{\tau^{c}}\left(f_{i} \mid i \in I\right)
$$

Поскольку $P=C^{p}(A)$ и $A \in \mathfrak{H}$, то

$$
A / P=A / C^{p}(A) \in h(p)=\left(\vee_{\omega_{\infty}}^{\tau^{c}}\left(f_{i} \mid i \in I\right)\right)(p)=\vee_{\omega_{\infty}}^{\tau}\left(f_{i}(p) \mid i \in I\right) .
$$

Так как $|A / P|<|A|$, то в силу выбора группы $A$ существует такой набор индексов $\hat{J}=\left\{j_{1}, \ldots, j_{n}\right\}$, что

$$
A / P \in \mathrm{V}_{\omega_{\infty}}^{\tau_{\infty}^{c}}\left(f_{j}(p) \mid j \in \hat{J}\right) .
$$

Вследствие леммы $2.10 w=\vee_{\omega_{\infty}}^{\tau^{c}}\left(f_{j} \mid j \in \hat{J}\right)$-минимальный $\omega$-композиционный $c_{\omega_{\infty}^{\tau}}^{\tau}$-значный спутник формации

$$
\mathfrak{W}=\vee_{\omega_{\infty}}^{\tau^{c}}\left(\mathfrak{F}_{j} \mid j \in \hat{J}\right)
$$

Следовательно,

$$
A / O_{p}(A)=A / P \in \vee_{\omega_{\infty}}^{\tau^{c}}\left(f_{j}(p) \mid j \in \hat{J}\right)=\left(\vee_{\omega_{\infty}}^{\tau^{c}}\left(f_{j} \mid j \in \hat{J}\right)\right)(p)=w(p) .
$$

Поскольку спутник $w$-внутренний, то, применяя лемму 1.8 , заключаем, что группа $A \in \mathfrak{W}$. Следовательно,

$$
\mathfrak{F}=c_{\omega_{\infty}}^{\tau} \text { form } A \subseteq \mathfrak{W}=\vee_{\omega_{\infty}}^{\tau^{c}}\left(\mathfrak{F}_{j} \mid j \in \hat{J}\right)
$$

Полученное противоречие показывает, что $\mathfrak{F}-$ компактный элемент решетки $c_{\omega_{\infty}}^{\tau}$. Поскольку любая $\tau$-замкнутая тотально $\omega$-композиционная формация является объединением своих однопорожденных $\tau$-замкнутых тотально $\omega$-композиционных подформаций в решетке $c_{\omega_{\infty}}^{\tau}$, то решетка $c_{\omega_{\infty}}^{\tau}$ алгебраична.

Заметим, что ввиду леммы 4.8.1 [Воробьев, 2012, с. 247] (см. также лемма 4.1(a) [Skiba, Vorob'ev, 2013]) компактными элементами решетки $c_{\omega_{\infty}}^{\tau}$ являются в точности однопорожденные формации из $c_{\omega_{\infty}}^{\tau}$

Поскольку каждая полная подрешетка алгебраической решетки формаций также является алгебраической решеткой (см. [Шербина, Сафонов, 2019б]), то из теоремы 3.1 получаем

Следствие 3.1. Каждая полная подрешетка решетки $c_{\omega_{\infty}}^{\tau}$ является алгебраической. 
Отметим также другие следствия доказанной теоремы. Следующее следствие является решением проблемы 1 [Скиба, Шеметков, 2000]. Если $\tau$-тривиальный подгрупповой функтор, то из теоремы 3.1 получаем

Следствие 3.2. Решетка $c_{\infty}^{\omega}$ всех тотально $\omega$-композиционных формаций является алгебраической.

В случае, когда $\tau(G)=\mathrm{S}_{n}(G)$ для любой группы $G$, из теоремы 3.1 имеем

Следствие 3.3. Решетка всех нормально наследственных тотально $\omega$-композиционных формаций является алгебраической.

Если $\omega=\{p\}$, то из теоремы 3.1 получаем

Следствие 3.4. Решетка $c_{p_{\infty}}^{\tau}$ всех $\tau$-замкнутых тотально $p$-композиционных формаций является алгебраической.

Если $\omega=\mathbb{P}$-множество всех простых чисел, то из теоремы 3.1 вытекает

Следствие 3.5. [Tsarev, 2019b]. Решетка $c_{\infty}^{\tau}$ всех $\tau$-замкнутых тотально композиционных формаций является алгебраической.

В случае $\omega=\mathbb{P}$ для тривиального подгруппового функтора $\tau$ из теоремы 3.1 вытекает

Следствие 3.6. [Tsarev, 2019a]. Решетка $c_{\infty}$ всех тотально композиционньх формаций является алгебраической.

Заключение. В работе получены положительные ответы двух открытых проблем теории решеток частично композиционных формаций: А. Н. Скибы и Л. А. Шеметкова об алгебраичности решетки всех (функторно замкнутых) частично тотально композиционных формаций, а также А. А. Царева и Н. Н. Воробьева об индуктивности указанной решетки. В качестве следствия основного результата установлены алгебраичность и индуктивность решетки $c_{p_{\infty}}^{\tau}$ всех $\tau$-замкнутых тотально $p$-композиционных формаций, а также решетки $c_{\infty}^{\tau}$ всех $\tau$-замкнутых тотально композиционных формаций. Аналогичные результаты получены для решеток функторно замкнутых частично тотально композиционных формаций, соответствующих некоторым подгрупповым функторам $\tau$. Тем самым найдены новые классы алгебраических и индуктивных решеток формаций. Полученные результаты могут быть использованы в исследованиях по теории решеток частично композиционных формаций конечных групп.

Благодарность. Автор выражает искреннюю признательность В. Г. Сафонову за полезные обсуждения работы.

\section{Список литературы}

1. Биркгоф Г. 1984. Теория решеток. Пер. с англ. М., Наука, 568 с.

2. Воробьев Н. Н. 2000. Об индуктивных решетках формаций и классов Фиттинга. Доклады НАН Беларуси, 44(3): 21-24.

3. Воробьев Н. Н. 2012. Алгебра классов конечных групп. Витебск, «Витебский государственный университет имени П. М. Машерова», 322 с.

4. Воробьев Н. Н., Царев А. А. 2010. О модулярности решетки $\tau$-замкнутых $n$-кратно $\omega$-композиционных формаций. Украинский математический журнал, 62(4): 453-463.

5. Жизневский П. А. 2010. О модулярности и индуктивности решетки всех $\tau$-замкнутых $n$-кратно $\omega$-композиционных формаций конечных групп. Известия Гомельского государственного университета имени Ф. Скорины, 58(1): 185-191.

6. Сафонов В. Г. 2004. О тотально $\omega$-насыщенных формациях конечных групп. Препринт, № 7 . Гомель, «Гомельский государственный университет им. Ф. Скорины», 18 с.

7. Сафонов В. Г. 2007. Характеризация разрешимых однопорожденных тотально насыщенных формаций конечных групп. Сибирский математический журнал, 48(1): 185-191. DOI: $10.1007 / \mathrm{s} 11202-007-0015-3$.

8. Сафонов В. Г. 2010. $\mathfrak{G}$-отделимость решетки $\tau$-замкнутых тотально насыщенных формаций. Алгебра и логика, 49(5): 690-702. DOI: 10.1007/s10469-010-9110-9.

9. Сафонов В. Г., Сафонова И. Н. 2014. О минимальных тотально $\omega$-насыщенных ненильпотентных формациях конечных групп. Вестник Витебского государственного университета, 84(6): 9-15.

10. Сафонов В. Г., Сафонова И. Н. 2017. Отделимость решетки $\tau$-замкнутых тотально $\omega$-насыщенных формаций конечных групп. Проблемы физики, математики и техники, 33(4): 76-83. 
11. Сафонов В. Г., Шеметков Л. А. 2008. О подрешетках решетки тотально насыщенных формаций конечных групп. Доклады НАН Беларуси, 52(4): 34-37.

12. Селькин В. М. 2011. Однопорожденные формации. Гомель, «Гомельский государственный университет имени Ф. Скорины», 2011, 240 с.

13. Скиба А. Н. 1986. О локальных формациях длины 5. В кн.: Арифметическое и подгрупповое строение конечных групп: труды Гомельского семинара. Под. ред. М. И. Салука. Минск, Наука и техника, 135-149.

14. Скиба А. Н. 1987. Характеризация конечных разрешимых групп заданной нильпотентной длины. В кн.: Вопросы алгебры. Вып. 3. Минск, «Университетское»: 21-31.

15. Скиба А. Н. 1997. Алгебра формаций. Минск, Беларуская навука, 240 с.

16. Скиба А. Н., Шеметков Л. А. 1999. Кратно $\omega$-локальные формации и классы Фиттинга конечных групп. Математические труды, 2(2): 114-147.

17. Скиба А. Н., Шеметков Л. А. 2000. Кратно $\mathfrak{L}-$ Композиционные формации конечных групп. Украинский математический журнал, 52(6): 783-797.

18. Шеметков Л. А. 1978. Формации конечных групп. М., Наука, 267 с.

19. Шеметков Л. А. 1984. О произведении формаций. Доклады АН БССР, 28(2): 101-103.

20. Шеметков Л. А, Скиба А. Н. 1989. Формации алгебраических систем. М., Наука, 253 с.

21. (а) Щербина В. В., Сафонов В. Г. 2019. О подрешетках решетки частично тотально насыщенных формаций конечных групп. Научные ведомости Белгородского государственного университета. Серия: Математика. Физика. 51(1): 64-87. DOI 10.18413/2075-4639-2019-51-1-64-87.

22. (б) Щербина В. В., Сафонов В. Г. 2019. О некоторых свойствах решетки частично тотально насыщенных формаций конечных групп. Научные ведомости Белгородского государственного университета. Серия: Математика. Физика. 51(2): 227-244. DOI 10.18413/2075-4639-2019-51-2227-244.

23. Ballester-Bolinches A., Ezquerro L. M. 2006. Classes of Finite Groups. Dordrecht, Springer, 385 p.

24. Doerk K., Hawkes T. 1992. Finite Soluble Groups. Berlin, New York, Walter de Gruyter \& Co, 891 p.

25. Gaschuts W. 1963. Zur Theorie der endlichen auflosbaren Gruppen [On the theory of finite soluble groups]. Mathematische Zeitschrift, 80(4): 300-305.

26. Guo W. 2000. The Theory of Classes of Groups. Beijing, New York, Dordrecht, Boston, London, Science Press, Kluwer Academic Publishers, 2000, 261 p.

27. Guo W., Sel'kin V.M., Shum K. P. 2007. Factorization theory of 1-generated $\omega$-composition formations. Communications in Algebra, 35(9): 2901-2931. DOI: 10.1080/00927870701302248.

28. Skiba A. N., Vorob'ev N. N. 2013. On the lattice of saturated and solubly saturated formations of finite groups. Southest Asian Bulletin of Mathematics, 37(5): 771-780.

29. Tsarev A. A. 2018. Inductive lattices of totally composition formations. Revista Colombiana de Matematicas, 52(2): 161-169. DOI: https://dx.doi.org/10.15446/recolma.v52n2.77156 (accessed: 6 November 2019).

30. (a) Tsarev A. A. 2019. On the lattice of all totally composition formations of finite groups. Ricerche di Matematica, 68(2): 693-698. DOI: 10.1007/s11587-019-00433-3.

31. (b) Tsarev A. A. 2019. On the lattice of all $\tau$-closed totally composition formations of finite groups. Materials of international conference «Algebra and Mathematical Logic: Theory and Applications». Kazan, Kazan (Volga Region) Federal University: 62-63.

32. Tsarev A. A., Vorob'ev N. N. 2018. Lattices of composition formations of finite groups and the laws. Journal of Algebra and Its Applications, 17(5): 1850084 (17 pages). DOI: 10.1142/S0219498818500846. 


\section{References}

1. Birkhoff G. 1984. Teoriya reshetok [Lattice Theory]. Moscow, Publ. Nauka, 568 p.

2. Vorob'ev N. N. 2000. Ob induktivnykh reshetkakh formatsiy i klassov Fittinga [On inductive lattices of formations and Fitting classes]. Doklady NAN Belarusi, 44(3): 21-24.

3. Vorob'ev N. N. 2012. Algebra klassov konechnykh grupp [Algebra of Classes of Finite Groups]. Vitebsk, Publ. «Vitebskiy gosudarstvennyy universitet imeni P. M. Masherova», 322 p.

4. Vorob'ev N. N., Tsarev A. A. 2010. O modulyarnosti reshetki $\tau$-zamknutykh $n$-kratno $\omega$-kompozitsionnykh formatsiy [On the modularity of a lattice of $\tau$-closed $n$-multiply $\omega$-composition formations]. Ukrainskiy matematicheskiy zhurnal, 62(4): 453-463.

5. Zhiznevsky P. A. 2010. O modulyarnosti i induktivnosti reshetki vsekh $\tau$-zamknutykh $n$-kratno $\omega$ kompozitsionnykh formatsiy konechnykh grupp [On modularity and inductance of the lattice of all $\tau$ closed $n$-multiply $\omega$-composition formations of finite groups]. Izvestiya Gomel'skogo gosudarst vennogo universiteta imeni F. Skoriny, 58(1): 185-191.

6. Safonov V. G. 2004. O total'no $\omega$-nasyshchennykh formatsiyakh konechnykh grupp. Preprint, № 7 [On totally $\omega$-saturated formations of finite groups. Preprint, No. 7]. Gomel, Publ. «Gomel'skiy gosudarstvennyy universitet im. F. Skoriny», 18 p.

7. Safonov V. G. 2007. Characterization of the soluble one-generated totally saturated formations of finite groups. Siberian Mathematical Journal, 48 (1): 185-191 (in Russian). DOI: 10.1007/s11202007-0015-3.

8. Safonov V. G. 2010. $\mathfrak{G}$-separability of the lattice of $\tau$-closed totally saturated formations. Algebra and Logic, 49(5): 690-702 (in Russian). DOI: 10.1007/s10469-010-9110-9.

9. Safonov V. G., Safonova I. N. 2014. O minimal'nykh total'no $\omega$-nasyshchennykh nenil'potentnykh formatsiyakh konechnykh grupp [On minimal totally $\omega$-saturated non-nilpotent formations of finite groups]. Vestnik Vitebskogo gosudarstvennogo universiteta, 84(6): 9-15.

10. Safonov V. G., Safonova I. N. 2017. Otdelimost' reshetki $\tau$-zamknutykh total'no $\omega$-nasyshchennykh formatsiy konechnykh grupp [Separability of the lattice of $\tau$-closed totally $\omega$-saturated formations of finite groups]. Problems of Physics, Mathematics and Technics, 33(4): 76-83.

11. Safonov V. G., Shemetkov L. A. 2008. O podreshetkakh reshetki total'no nasyshchennykh formatsiy konechnykh grupp [Sublattices of the lattice of totally saturated formations of finite groups]. Doklady NAN Belarusi, 52(4): 34-37.

12. Sel'kin V. M. 2011. Odnoporozhdennye formatsii [One-generated formations]. Gomel, Publ. «Gomel'skiy gosudarstvennyy universitet imeni F. Skoriny», 2011, 240 p.

13. Skiba A. N. 1986. O lokal'nykh formatsiyakh dliny 5 [On local formations of length 5]. V kn.: Arifmeticheskoe i podgruppovoe stroenie konechnykh grupp: trudy Gomel'skog seminara [Arithmetic and subgroup structure of finite groups: Gomel seminar proceedings]. Pod. red. M. I. Saluka. Minsk, Publ. Nauka i tekhnika: $135-149$.

14. Skiba A. N. 1987. Kharakterizatsiya konechnykh razreshimykh grupp zadannoy nil'potentnoy dliny [Characterization of finite soluble groups with given nilpotent length]. V kn.: Voprosy algebry. Vyp. 3 [Algebra Problems. Vol. 3]. Minsk, Publ. «Universitetskoe»: 21-31.

15. Skiba A. N. 1997. Algebra formatsiy [Algebra of Formations]. Minsk, Belaruskaya navuka, 240 p.

16. Skiba A. N., Shemetkov L. A. 1999. Multiply $\omega$-local formations and Fitting classes of finite groups. Mathematical Proceedings, 2(2): 114-147 (in Russian).

17. Skiba A. N., Shemetkov L.A. 2000. Kratno $\mathfrak{L}$-kompozitsionnye formatsii konechnykh grupp [Multiply $\mathfrak{L}$-composition formations of finite groups]. Ukrainskiy matematicheskiy zhurnal, 52(6): 783-797.

18. Shemetkov L. A. 1978. Formatsii konechnykh grupp [Formations of Finite Groups]. Moscow, Publ. Nauka, $267 \mathrm{p}$.

19. Шеметков Л. А. 1984. O proizvedenii formatsiy [On product of formations]. Doklady AN BSSR, 28(2): 101-103. 
20. Shemetkov L. A, Skiba A. N. 1989. Formatsii algebraicheskikh sistem [Formations of Algebraic Systems]. Moscow, Publ. Nauka, 253 p.

21. Shcherbina V. V., Safonov V. G. 2019. On sublattices of the lattice of partially totally saturated formations of finite groups. Belgorod State University Scientific Bulletin. Mathematics. Physics. 51(1): 64-87 (in Russian). DOI 10.18413/2075-4639-2019-51-1-64-87.

22. Shcherbina V. V., Safonov V. G. 2019. On some properties of the lattice of partially totally saturated formations of finite groups. Belgorod State University Scientific Bulletin. Mathematics. Physics. 51(2): 227-244 (in Russian). DOI 10.18413/2075-4639-2019-51-2-227-244.

23. Ballester-Bolinches A., Ezquerro L. M. 2006. Classes of Finite Groups. Dordrecht, Springer, 2006, $385 \mathrm{p}$.

24. Doerk K., Hawkes T. 1992. Finite Soluble Groups. Berlin, New York, Walter de Gruyter \& Co, 891 p.

25. Gaschuts W. 1963. Zur Theorie der endlichen auflosbaren Gruppen [On the theory of finite soluble groups]. Mathematische Zeitschrift, 80(4): 300-305.

26. Guo W. 2000. The Theory of Classes of Groups. Beijing, New York, Dordrecht, Boston, London, Science Press, Kluwer Academic Publishers, 2000, 261 p.

27. Guo W., Sel'kin V.M., Shum K.P. 2007. Factorization theory of 1-generated $\omega$-composition formations. Communications in Algebra, 35(9): 2901-2931. DOI: 10.1080/00927870701302248.

28. Skiba A. N., Vorob'ev N. N. 2013. On the lattice of saturated and solubly saturated formations of finite groups. Southest Asian Bulletin of Mathematics, 37(5): 771-780.

29. Tsarev A. A. 2018. Inductive lattices of totally composition formations. Revista Colombiana de Matematicas, 52(2): 161-169. DOI: https://dx.doi.org/10.15446/recolma.v52n2.77156 (accessed: 6 November 2019).

30. (a) Tsarev A. A. 2019. On the lattice of all totally composition formations of finite groups. Ricerche di Matematica, 68(2): 693-698. DOI: 10.1007/s11587-019-00433-3.

31. (b) Tsarev A. A. 2019. On the lattice of all $\tau$-closed totally composition formations of finite groups. Materials of international conference «Algebra and Mathematical Logic: Theory and Applications». Kazan, Kazan (Volga Region) Federal University: 62-63.

32. Tsarev A. A., Vorob'ev N. N. 2018. Lattices of composition formations of finite groups and the laws. Journal of Algebra and Its Applications, 17(5): 1850084 (17 pages). DOI: 10.1142/S0219498818500846.

Щербина Владимир Владимирович - магистрант Государственного учреждения образования «Институт подготовки научных кадров Национальной академии наук Беларуси» ул. Кнорина, 1, г. Минск, Республика Беларусь, 220049

E-mail: shcherbinavv@tut.by 